\title{
An Isoform-Specific Mutant Reveals a Role of PDP1 $\varepsilon$ in the Circadian Oscillator
}

\author{
Xiangzhong Zheng, ${ }^{1 \star}$ Kyunghee Koh, ${ }^{1 \star}$ Mallory Sowcik, ${ }^{2}$ Corinne J. Smith, ${ }^{2}$ Dechun Chen, ${ }^{1}$ Mark N. Wu, ${ }^{3}$ \\ and Amita Sehgal ${ }^{1,2}$ \\ ${ }^{1}$ Department of Neuroscience, ${ }^{2}$ Howard Hughes Medical Institute, and ${ }^{3}$ Department of Neurology, Division of Sleep Medicine, University of Pennsylvania, \\ Philadelphia, Pennsylvania 19104
}

The Drosophila PAR domain protein 1 (Pdp1) gene encodes a transcription factor with multiple functions. One isoform, PDP1 1 , was proposed to be an essential activator of the core clock gene, Clock (Clk). However, a central clock function for PDP1 $\varepsilon$ was recently disputed, and genetic analysis has been difficult due to developmental lethality of Pdp1-null mutants. Here we report the discovery of a mutation that specifically disrupts the $P d p 1 \varepsilon$ isoform. Homozygous $P d p 1 \varepsilon$ mutants are viable and exhibit arrhythmic circadian behavior in constant darkness and also in the presence of light:dark cycles. Importantly, the mutants show diminished expression of CLK and PERIOD (PER) in the central clock cells. In addition, expression of PDF (pigment-dispersing factor) is reduced in a subset of the central clock cells. Loss of Pdp1E also alters the phosphorylation status of the CLK protein and disrupts cyclic expression of a per-luciferase reporter in peripheral clocks under free-running conditions. Transgenic expression of PDP1 $1 \varepsilon$ in clock neurons of $P d p 1 \varepsilon$ mutants can restore rhythmic circadian behavior. However, transgenic expression of CLK in these mutants rescues the expression of PER in the central clock, but fails to restore behavioral rhythms, suggesting that PDP1 $\varepsilon$ has effects outside the core molecular clock. Together, these data support a model in which PDP1 $\varepsilon$ functions in the central circadian oscillator as well as in the output pathway.

\section{Introduction}

The molecular circadian clock consists of interlocking feedback loops in which clock gene products regulate their own expression (Hardin, 2005). In one feedback loop in Drosophila, the circadian clock genes period (per) and timeless (tim) are activated by CLOCK-CYCLE (CLK-CYC) heterodimers during the late day, and their transcripts peak in the early night. Later in the night, the PER and TIM proteins translocate into the nucleus and inhibit CLK-CYC activity, thus repressing per and tim transcription. In a second feedback loop, CLK-CYC activate the basic leucine zipper transcription factors Pdp1 $\varepsilon$ and vrille (vri). Both PDP $1 \varepsilon$ and VRI proteins can bind to $\mathrm{E} 4 \mathrm{BP} 4$ sites in the $\mathrm{Clk}$ promoter and activate or repress $\mathrm{Clk}$ transcription, respectively. PDP $1 \varepsilon$ expression lags behind that of VRI, and the sequential repression and activation may be the driving force behind rhythmic Clk expression (Cyran et al., 2003). This feedback loop also regulates overall Clk levels, so that overexpression of vri (Glossop et al., 2003) or reduced expression of $P d p 1$ results in reduced Clk expression (Cyran et al., 2003).

\footnotetext{
Received May 6, 2009; revised July 17, 2009; accepted July 20, 2009.

This work was supported by National Institute of Neurological Disorders and Stroke (NINDS) Grant R01NS048471 (A.S.), a National Alliance for Research on Schizophrenia and Depression Young Investigator award (K.K.), a Career Award for Medical Scientists Award from the Burroughs-Wellcome Foundation (M.N.W.), and NINDS Grant K08NS059671 (M.N.W.). A.S. is an investigator of the Howard Hughes Medical Institute. We thank Isaac Edery (Rutgers University), Paul Hardin (University of Houston), Ravi Allada (Northwestern University), Paul Taghert (Washington University in St. Louis), and Justin Blau (New York University) for providing fly stocks and antibodies. We are especially thankful to Andrea Lougheed, Robert Hardy, and Charles Zuker for providing Zuker collection mutants for screening.

*X.Z. and K.K. contributed equally to this work.

Correspondence should be addressed to Amita Sehgal, 232 Stemmler Hall, Department of Neuroscience, University of Pennsylvania Medical School, Philadelphia, PA 19104. E-mail: amita@mail.med.upenn.edu.

D0I:10.1523/JNEUROSCI.2133-09.2009

Copyright $\odot 2009$ Society for Neuroscience $\quad$ 0270-6474/09/2910920-08\$15.00/0
}

$P d p 1$ has seven isoforms, one of which, $P d p 1 \varepsilon$, exhibits rhythmic expression (Cyran et al., 2003) and is thought to function in the circadian clock. A null mutation (P205) that removes all Pdp1 isoforms causes developmental lethality (Reddy et al., 2006) but shows dramatically reduced Clk levels in larvae (Cyran et al., 2003), consistent with cell culture data demonstrating that PDP1 can activate $\mathrm{Clk}$ transcription. However, two recent studies challenged the requirement of PDP1 in the molecular circadian oscillator (Benito et al., 2007). Overexpression of Pdp1 $1 \varepsilon$ or RNAi knock down of $P d p 1$ in clock cells disrupted circadian locomotor rhythms without affecting expression of $\mathrm{Clk}$ or other clock genes (Benito et al., 2007). Similar results were obtained when PDP1 activity was inhibited by transgenic expression of a dominantnegative form of PDP1 (Lim et al., 2007). Based on these results, it was suggested that PDP1 functions in the circadian output pathway rather than as an essential component of the circadian oscillator. The reduction of $\mathrm{Clk}$ expression in Pdp1-null mutant larvae was attributed to developmental defects. However, RNAi is unlikely to completely eliminate PDP1 activity $[\sim 70 \%$ reduction by RNAi was reported in Benito et al. (2007)], and the residual PDP1 activity may be sufficient for normal molecular cycling, but not for regulation of downstream output genes. In addition, both RNAi and dominant-negative PDP1 indiscriminately affect all isoforms of $P d p 1$. Thus it remains unclear whether PDP1 $\varepsilon$ has a role in the circadian feedback loop of the adult fly as an essential positive regulator of $C l k$ transcription.

Through a forward-genetic screen of EMS-mutagenized lines for abnormal circadian sleep:wake behavior (Wu et al., 2008), we identified a mutation that specifically disrupts the Pdple isoform. Loss of $P d p 1 \varepsilon$ is not associated with any lethality, but causes arrhythmic circadian locomotor behavior. More importantly, we show here 

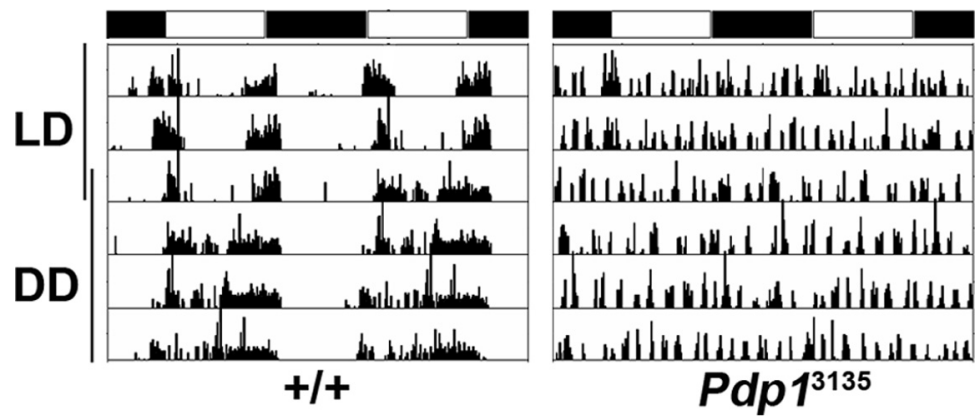

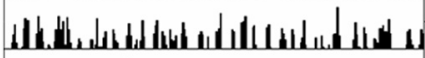

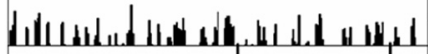

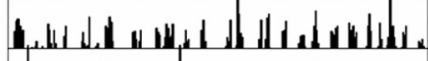

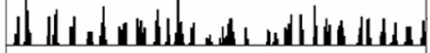

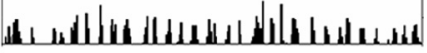
Pdp13135
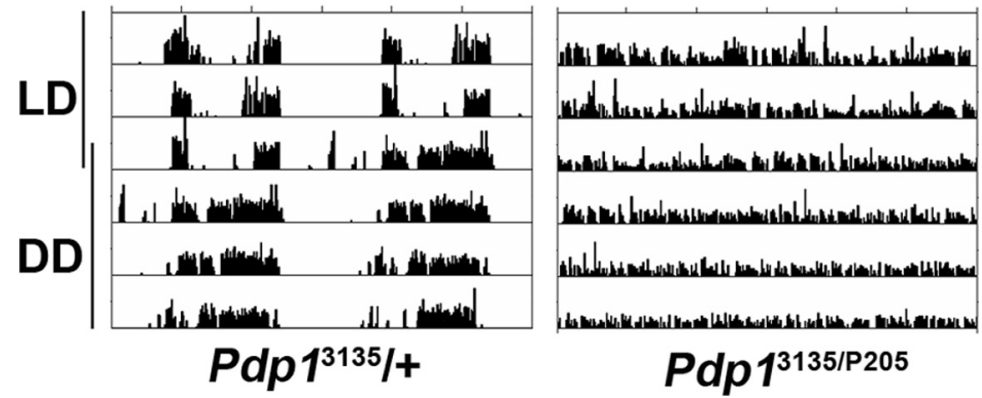

Figure 1. Circadian locomotor activities of $P d p 1$ mutant flies. While control flies are rhythmic, $P d p 1^{3135}$ mutant flies, as homozygotes and transheterozygotes over the null allele $P d p 1^{P 205}$, are arrhythmic under LD and DD conditions. Heterozygous 3135 flies are rhythmic, indicating that the mutation is recessive.

Table 1. Free-running circadian locomotor rhythm phenotypes of Pdp1 mutants and flies overexpressing UAS-Pdp1

\begin{tabular}{|c|c|c|c|}
\hline Genotype & $n$ & $R \%$ & Period $(h) \pm$ SEM \\
\hline$w / Y ;+;+$ & 48 & 98 & $23.63 \pm 0.030$ \\
\hline$w / Y ; ; P d p 1^{3135} / \mathrm{TM} 6 \mathrm{~B}$ & 61 & 98 & $23.55 \pm 0.036$ \\
\hline$w / Y ; ; P d p 1^{3135}$ & 48 & 0 & \\
\hline$w / Y ; ; P d p 1^{3135} / \mathrm{Df}(3 \mathrm{~L})-\mathrm{RM} 5-2$ & 30 & 0 & \\
\hline$w / Y ; ; P d p 1^{3135} / D f(3 L)-p b l-X 1$ & 23 & 0 & \\
\hline$w / Y ; ; P d p 1^{3135 / P 205}$ & 31 & 0 & \\
\hline$w / Y_{; \prime} \cdot P d p 1^{3135} / C l k^{\text {jrk }}$ & 32 & 84 & $23.79 \pm 0.100$ \\
\hline$w / N_{;} ; P d p 1^{3135} / C l k^{\text {ar }}$ & 19 & 100 & $23.90 \pm 0.071$ \\
\hline$w / Y ; ; P d p 1^{\mathrm{d} 11071} /+$ (7 d old $)$ & 48 & 98 & $23.63 \pm 0.060$ \\
\hline$w / Y_{; \prime}: P d p 1^{\mathrm{d} 11071 / 3135}$ (7 d old $)$ & 81 & 84 & $23.88 \pm 0.062$ \\
\hline$w / Y ; ; P d p 1^{\mathrm{d} 11071}(7 \mathrm{~d}$ old $)$ & 60 & 22 & $23.44 \pm 0.384$ \\
\hline$w / Y \because ; P d p 1^{\mathrm{d} 11071} /+(25 \mathrm{~d}$ old $)$ & 32 & 100 & $24.09 \pm 0.085$ \\
\hline$w / Y ; ; P d p 1^{\mathrm{d} 11071 / 3135}$ (25 d old) & 27 & 59 & $24.15 \pm 0.159$ \\
\hline$w / Y ; T U G /+; P d p 1^{3135}$ & 9 & 0 & \\
\hline$w / Y ; T U G / U A S-P d p 1 ; P d p 1^{3135}$ & 28 & 96 & $23.94 \pm 0.132$ \\
\hline$w / Y ; T U G / U A S-P d p 1 ; P d p 1^{3135 / P 205}$ & 27 & 96 & $23.77 \pm 0.084$ \\
\hline cry24-G/Y;:Pdp $1^{3135}$ & 41 & 0 & \\
\hline cry24-G/Y;UAS-Pdp1;Pdp1 3135 & 14 & 93 & $23.69 \pm 0.165$ \\
\hline$w / Y ; P d f-G /+; P d p 1^{3135}$ & 10 & 0 & \\
\hline$w / Y ; P d f-G / U A S-P d p 1 ; P d p 1^{3135}$ & 30 & 0 & \\
\hline$w / Y ; T U G /+;$ & 32 & 100 & $23.55 \pm 0.035$ \\
\hline$w / Y ; T U G / U A S-P d p 1 ;$ & 32 & 97 & $23.74 \pm 0.113$ \\
\hline$w / Y ; P d f-G /+$ & 29 & 100 & $24.21 \pm 0.058$ \\
\hline$w / Y ; P d f-G / U A S-P d p 1$ & 32 & 100 & $24.36 \pm 0.108$ \\
\hline$y w / Y ; U A S-P d p 1 / C y 0$ & 39 & 100 & $23.77 \pm 0.021$ \\
\hline$y w / Y ; T G / U A S-P d p 1$ & 27 & 100 & $24.25 \pm 0.061$ \\
\hline
\end{tabular}

w: $w^{1118} ; y w: y^{1}, w^{1118} ;$ TUG: tim-(UAS)-Gal4; cry24-G: cry-Gal4 (line 24); Pdf-G: Pdf-Gal4.

that expression of circadian clock proteins CLK and PER is substantially reduced in the clock neurons of $P d p 1 \varepsilon$ mutants. In addition, expression of pigment-dispersing factor (PDF) in some clock cells is reduced in $P d p 1 \varepsilon$ mutants. Transgenic expression of CLK in $P d p 1 \varepsilon$ mutants rescues the cyclic expression of PER in clock cells, but fails to restore behavioral rhythms. These data demonstrate that PDP1ع is required as an activator of $C l k$ as well as a regulator of circadian output.

\section{Materials and Methods}

Drosophila genetics. An arrhythmic line (Z23135) was identified in a screen of EMSmutagenized lines from the Zuker collection (Koundakjian et al., 2004; Wu et al., 2008). Initial mapping revealed that the mutation causing arrhythmia was on the third chromosome even though the line was balanced on the second chromosome. For meiotic recombination mapping, we crossed Roughened $(R)$ Dichaete $(D) / 3135$ females to Z2-3135 homozygous males. Of $53 R$ not- $D$ progeny, 27 were arrhythmic, and of 76 not- $R, D$ progeny, 29 were arrhythmic. This placed the mutation approximately at cytological location 66. Complementation tests with deficiencies and candidate genes suggested that the mutation lies in the $P d p 1$ locus. Through sequencing, we identified a 4 bp deletion in an exon common to the Pdp1 RD and RJ isoforms. Next we outcrossed the mutant chromosome to an isogenic $w^{1118}$ background (iso31) for five generations, allowing recombination, and the molecular lesion was followed by PCR and sequencing. This mutation is referred to as $P d p 1^{3135}$ in this study.

A full-length $P d p 1-R D$ cDNA clone (GH27708) was obtained from DGRC, and the coding region was amplified by PCR using the following primers: 5'-GTT GCG GCC GCC AGT GAT CAT GTC GTC-3' and 5'-TGT CTC GAG ATC ATT ACA CAT CTT GGA A-3'. The PCR fragment was digested with NotI and XhoI, and inserted into the pUAST transformation vector. Transgenic lines carrying the UAS- $P d p 1-R D$ construct were generated using standard techniques (Rubin and Spradling, 1982 ) in an isogenic $w^{1118}$ (iso31) background (Rainbow Transgenic Flies), and two independent lines were used for rescue and overexpression experiments.

Circadian behavioral assays. Stocks were raised on standard food containing cornmeal, molasses, and yeast. Three- to five-day-old adult male flies were entrained to a $12 \mathrm{~h}: 12 \mathrm{~h}$ light:dark (LD) schedule at $25^{\circ} \mathrm{C}$ for $3 \mathrm{~d}$, and then loaded into locomotor assay tubes containing $5 \%$ sucrose and $2 \%$ agarose. Activity records collected under LD and constant darkness (DD) conditions were analyzed by using Clocklab software (Actimetrics). Circadian periodicity was evaluated using $\chi^{2}$ periodogram analysis. In the aging experiment, 2 - to 3 -d-old adult males were collected and maintained on standard food for $22 \mathrm{~d}$ at room temperature $\left(23^{\circ} \mathrm{C}\right)$ with change of food every $4 \mathrm{~d}$, and then entrained to an $\mathrm{LD}$ cycle at $25^{\circ} \mathrm{C}$ for $3 \mathrm{~d}$ and analyzed as described above.

Whole-fly per-luciferase reporter assay. Three- to five-day-old adult males were collected and entrained to a $12 \mathrm{~h}: 12 \mathrm{~h} \mathrm{LD}$ cycle at $25^{\circ} \mathrm{C}$ for $3 \mathrm{~d}$ on standard food, then loaded into Optiplate-96F plates (PerkinElmer) containing $100 \mu \mathrm{M}$ firefly luciferin (Biosynth), 5\% sucrose, and 2\% agar. Data were collected in DD over $5 \mathrm{~d}$ in a Topcount NXT luminometer (Packard). Each well was read twice, for $6 \mathrm{~s}$ each time, in a 30 min interval. Raw data were imported and analyzed in Excel.

Western blot analysis. Three- to five-day-old adult flies were entrained to $12 \mathrm{~h}: 12 \mathrm{~h} \mathrm{LD}$ cycles for $3 \mathrm{~d}$, and heads were collected at indicated time points for protein extraction. For DD samples, flies were collected on the second day in constant darkness, and Western blot analysis was performed as described previously (Sathyanarayanan et al., 2004). Guinea pig anti-PER, guinea pig anti-VRI (Glossop et al., 2003), guinea pig antiCLK (Lee et al., 1998), and rabbit anti-PDP1 (Cyran et al., 2003) were used at 1:3000 dilutions. Following enhanced chemiluminescence, images were taken in a Kodak image station or exposed to film, and images were processed using Adobe Photoshop.

Quantitative real-time PCR. Three- to five-day-old adult flies were maintained at a $12 \mathrm{~h}: 12 \mathrm{~h}$ light:dark cycle at $25^{\circ} \mathrm{C}$ for $3 \mathrm{~d}$ and then 


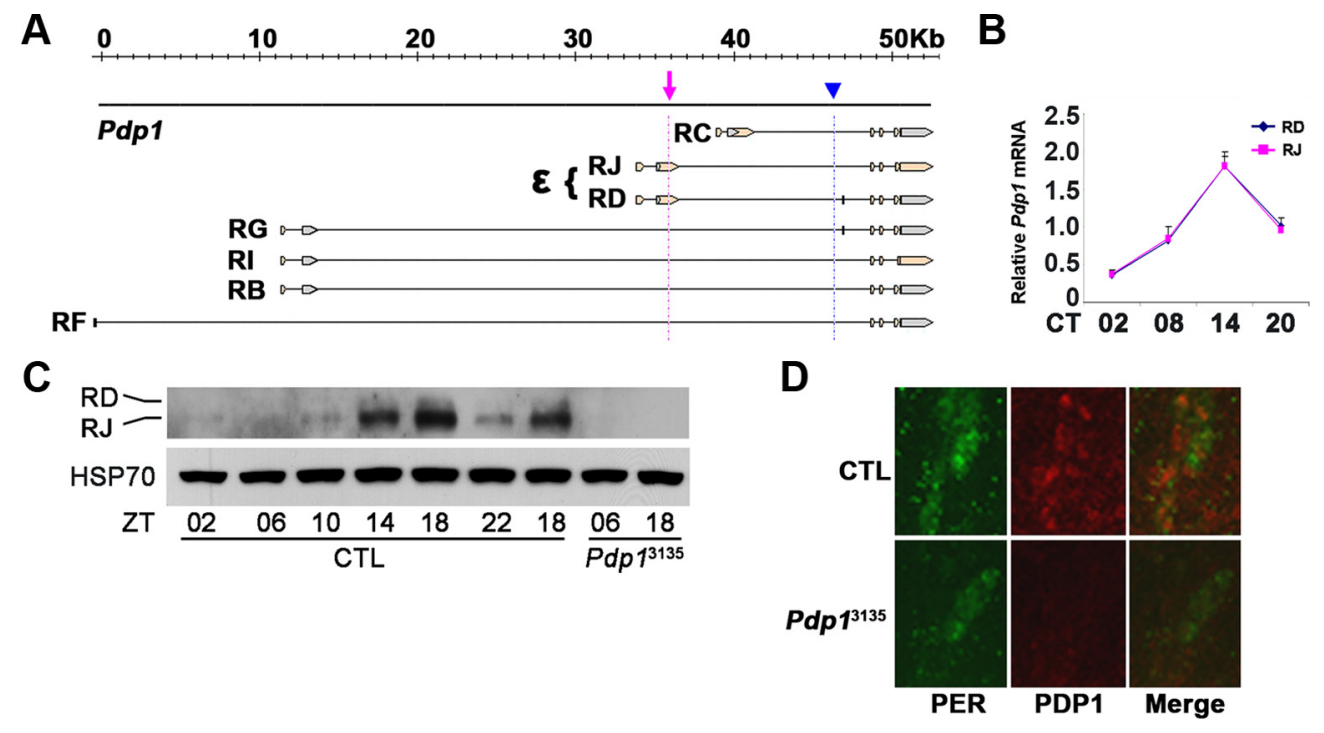

Figure 2. Molecular characterization of the $P d p 1^{3135}$ allele. $A, P d p 1$ has seven isoforms as annotated by Flybase. The RJ and RD isoforms are collectively referred to as the $\varepsilon$ isoform in this study. The only difference between $\mathrm{RJ}$ and $\mathrm{RD}$ is a small 42 bp exon (supplemental Fig. $1 B$, available at www.jneurosci.org as supplemental material). The $P d p 1^{3135}$ mutation has a $4 \mathrm{bp} \mathrm{deletion} \mathrm{(indicated}$ by an arrow) in the coding region of the second exon of RJ and RD. The triangle denotes a P-element insertion (d11071) in an intron common to all isoforms. $B$, mRNA levels of both the RD and RJ isoforms cycle in a circadian manner. Pdp $1 \mathrm{mRNA}$ levels were normalized to Actin mRNA levels. Data from four independent experiments for RJ and RD are shown. C, The RJ isoform, which is smaller than the RD isoform, is the predominant form in head extracts of control (CTL) wild-type flies. The secondZT18 sample is from the same wild-type strain carrying a UAS-Pdp 1 transgene; the transgene is not expressed due to lack of a Gal4 driver. Protein levels of both RD and RJ are undetectable in Pdp $1^{3135}$ mutant flies. D, Compared with wild-type controls, PDP1 expression at ZT20 is markedly reduced in the lateral neurons of $P d p 1^{3135}$ mutants. CTL, Background control for $P d p 1^{3135}$.

collected on dry ice at indicated time points on the third day of $\mathrm{LD}$ or the second day of DD. Total RNA was isolated using an Ultraspec RNA isolation system (Biotecx), and cDNAs were synthesized by using a highcapacity cDNA Archive kit (Applied Biosystems). Quantitative realtime PCR was performed in an ABI prism 7100 using a SYBR Green kit (Applied Biosystems).

Immunohistochemistry and microscopy. Fly brains were dissected in $4 \%$ PFA, washed for $1 \mathrm{~h}$ in PBS buffer, and incubated with primary antibody (in PBS buffer with 3\% normal donkey serum and 0.3\% Triton X-100) overnight at $4^{\circ} \mathrm{C}$. Brain samples were extensively washed with PBS buffer and incubated with Cy3 donkey anti-rat (or guinea pig) and FITC or Cy3 donkey anti rabbit secondary antibodies (Jackson ImmunoResearch Laboratories) for $1.5 \mathrm{~h}$ at room temperature, followed by extensive washes in PBS. Fluorescent images were taken with a Leica TCS SP5 confocal micro scope. Primary antibody dilutions were as follows: PER, 1:1500; PDF, 1:1000; and CLK (Houl et al., 2006), 1:500. Secondary antibody dilutions were 1:1000.

\section{Results}

A new allele of Pdp1 has arrhythmic circadian behavior

To isolate novel circadian mutants, we screened the Zuker collection of EMS-induced mutations (Koundakjian et al., 2004) for failure to show anticipatory locomotor behavior before lightdark transitions, as previously described (Wu et al., 2008). One of these lines (Z2-3135) exhibits arrhythmic behavior in both LD and DD conditions (Fig. 1). Through meiotic recombination and deficiency mapping, we found that this line carries a mutation that lies at the cytological position 66 on the third chromosome (see Materials and Methods). Since this region contains circadian clock genes $C l k$ and $P d p 1$, we suspected that one of them is mutated in Z2-3135. Complementation tests with Clk and Pdp1 alleles indicated that the new mutation (hereafter referred to as $P d p 1^{3135}$ ) maps to the $P d p 1$ locus (Fig. 1, Table 1).

\section{Molecular characterization of the $P d p 1 \varepsilon$ mutation}

Multiple promoters and alternative splicing events produce several Pdp1 isoforms (Reddy et al., 2000): there are seven annotated isoforms in Flybase (www.flybase.org) (Fig. 2A). To identify the molecular lesion of the new $P d p 1^{3135}$ allele, we sequenced the coding region of all $P d p 1$ isoforms. A 4 bp deletion was found in the second exon of the RD and RJ isoforms (Fig. $2 A$; supplemental Fig. $1 A$, available at www.jneurosci.org as supplemental material). While the Pdp1 $\varepsilon$ sequence reported in Cyran et al. (2003) matches that of the RD isoform, the sequence provided by Reddy et al. (2000) is that of the RJ isoform. RD and RJ isoforms differ by one small exon coding for 14 aa (supplemental Fig. $1 B$, available at www.jneurosci.org as supplemental material). The two isoforms share the same promoter region and transcriptional start site, and thus are likely to be regulated in a similar manner. Indeed, we find that transcript and protein levels of both isoforms are regulated in a circadian manner (Fig. $2 B, C$ ). We refer to RJ and RD collectively as the $\varepsilon$ isoform in this study.

The 4 bp deletion in $P d p 1^{3135}$ mutants affects both the RD and $\mathrm{RJ}$ isoforms but none of the other isoforms. The deletion results in a frame shift starting from residue 262 and a predicted truncated polypeptide of 374 aa (supplemental Fig. $1 A$, available at www.jneurosci.org as supplemental material), which is expected to be nonfunctional. Western analysis confirmed that PDP1 $\varepsilon$ is undetectable in $P d p 1^{3135}$ mutants (Fig. 2C). Therefore, the 3135 mutation is presumed to abolish the $\varepsilon$ isoform, but leave other isoforms intact.

Pdple is the dominant but not the only isoform that regulates circadian rhythms

$P d p 1^{\mathrm{P} 205}$-null mutants are lethal, and flies expressing a dominantnegative form of $P d p 1\left(P d p 1^{\mathrm{DN}}\right)$ and $P d p 1^{\mathrm{RNAi}}$ flies show anatomical abnormalities in the clock neurons (Lim et al., 2007), underscoring the important role of PDP1 in development. It is likely, however, that spatial and temporal expression patterns of different isoforms of $P d p 1$ are differentially regulated. $P d p 1^{3135}$ mutants are viable, which suggests that the PDP1 1 isoform does not play a significant developmental role. In addition, only the $P d p 1 \varepsilon$ isoform displays robust cycling at the mRNA and protein levels (Cyran et al., 2003). An antibody raised against the common region of the PDP1 isoforms detected robust cycling in the 
A
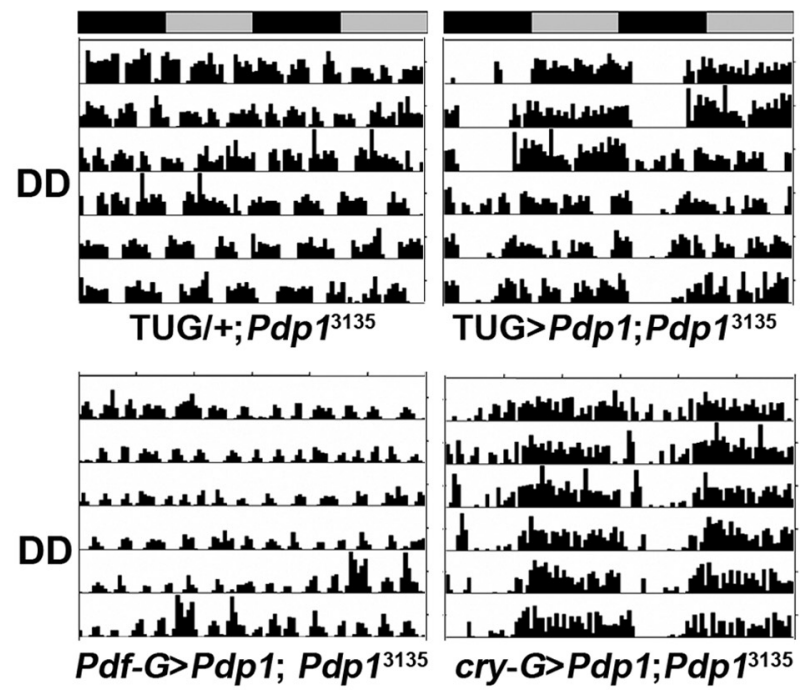

B
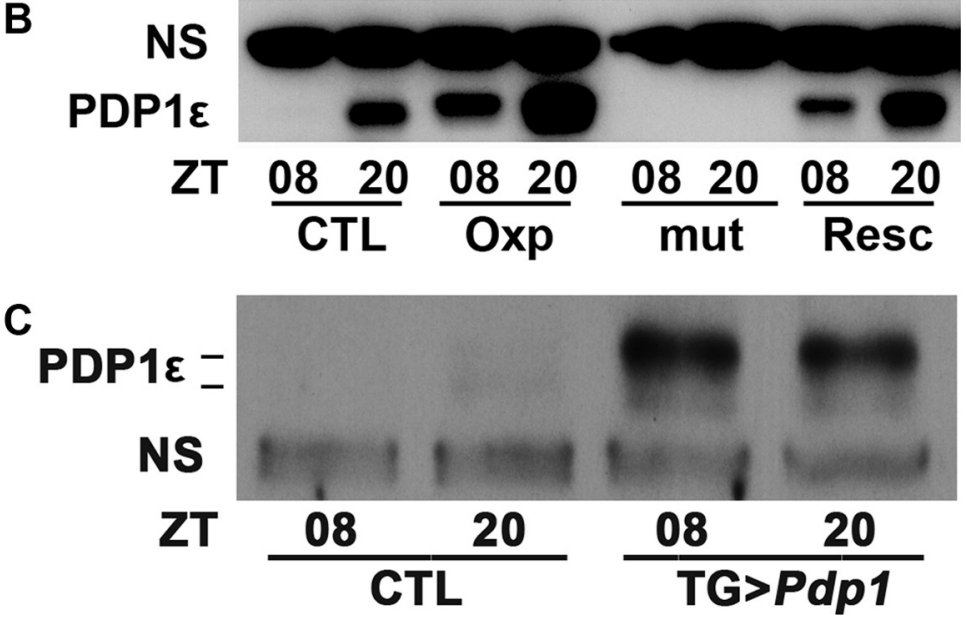

Figure 3. Rescue of $P d p 1^{3135}$ mutants using a UAS-Pdp1 transgene. $A$, Expression of a UAS-Pdp1-RD transgene rescued arrhythmic behavior when driven by tim-UAS-Gal4 (TUG) or cry-Gal4, but not by Pdf-Gal4. B, A UAS-Pdp1-RD transgene under the control of the TUG driver restores PDP1 $\varepsilon$ expression in $P d p 1^{3135}$ mutants (Resc). PDP1 $\varepsilon$ expression is increased above the normal levels when the transgene is driven by TUG in a wild-type background (0xp). PDP1 $\varepsilon$ levels cycle even when overexpressed. CTL, $\mathrm{TUG} /+;$ mut, $P d p 1^{3135}$.C, PDP1 protein levels do not cycle when overexpressed at a high level with a stronger promoter (tim-Gal4, TG). CTL, UAS-PdP1-RD/+.

central clock cells in the larval brain (Cyran et al., 2003), suggesting that PDP1 $\varepsilon$ is the major isoform in these clock cells. Using the same antibody, we found that PDP1 immunoreactivity in the central pacemaker cells of the adult brain is markedly reduced in $P d p 1^{3135}$ mutants (Fig. 2D), confirming that PDP $1 \varepsilon$ is indeed the predominant isoform expressed in the central circadian pacemaker.

We also identified a second mutation in $P d p 1, \mathrm{~d} 11071$, which is caused by a P-element insertion in an intron common to all isoforms of Pdp1 (Fig. 2A). The P-element carries a splicing acceptor, and thus is predicted to disrupt splicing of all $P d p 1$ isoforms. PDP $1 \varepsilon$ levels are markedly reduced, but detectable, in $P d p 1^{\mathrm{d} 11071}$ homozygous mutants, indicating that $\mathrm{d} 11071$ is a hypomorphic allele (supplemental Fig. 1C, available at www. jneurosci.org as supplemental material). Most (78\%) $P d p 1^{\text {d11071 ho- }}$ mozygous flies exhibit arrhythmic behavior (Table 1). Notably, $P d p 1^{\mathrm{d} 11071 / 3135}$ transheterozygous flies show a milder phenotype, which becomes more severe with aging; $\sim 26 \%$ of the 7 -d-old and $\sim 41 \%$ of the 25 -d-old transheterozygotes are arrhythmic. Our finding that $P d p 1^{\mathrm{d} 11071 / 3135}$ transheterozygous flies have less PDP1 $\varepsilon$ than
Pdp1 $1^{\mathrm{d} 11071}$ flies (supplemental Fig. $1 D$, available at www.jneurosci.org as supplemental material), but have a milder phenotype, suggests that PDP1e is not the only PDP1 isoform contributing to behavioral rhythms.

Transgenic expression of $P d p 1 \varepsilon$ restores behavioral rhythms

To confirm that the arrhythmic phenotype of $P d p 1^{3135}$ mutants maps to $P d p 1 \varepsilon$, we generated transgenic fly lines carrying a UAS-Pdp1-RD construct. Expression of wild-type $P d p 1-R D$ in the clock cells under the control of tim-UAS-Gal4 (TUG) or cry-Gal4 fully rescues the mutant phenotype (Table 1, Fig. 3A). Notably, the RJ isoform is more abundant than the RD isoform in wild-type flies (Fig. 2C), and thus our rescue result suggests that the RD and $\mathrm{RJ}$ isoforms are functionally interchangeable. On the other hand, expression of Pdp1-RD in PDF-expressing cells using the Pdf-Gal4 driver fails to rescue the arrhythmic behavior, probably due to its restricted expression pattern. We note that expression of UAS-Clk or UAS-cyc by a Pdf-Gal4 driver is also ineffective in rescuing $C l k^{\text {ar }}$ or $c y c{ }^{01}$ mutants (Allada et al., 2003; Peng et al., 2003). Overexpression of $P d p 1 \varepsilon$ in a wild-type background does not affect rhythmic behavior or free-running period (Table 1). Western blot analysis of head extracts confirmed that the UAS$P d p 1-R D$ transgene produces protein of the expected size whose abundance exhibits circadian cycling even when overexpressed by the TUG driver (Fig. 3B). However, when overexpressed with another, possibly stronger driver (tim-Gal4), PDP1 protein cycling is abolished (Fig. 3C). Interestingly, the behavioral rhythms of flies overexpressing PDP1 with tim-Gal4 are still unaffected (Table 1). These data suggest that cycling of PDP1 protein levels is not required for behavioral rhythms.

PDP1 $\varepsilon$ regulates expression of clock proteins in the central clock

Isolation of an isoform-specific mutant that specifically abolishes the $\varepsilon$ isoform of $P d p 1$ provides us with a unique opportunity to ascertain the role of PDP $1 \varepsilon$ in adult clock neurons. We first asked whether $P d p 1 \varepsilon$ plays a role in regulating CLK expression in the adult central clock cells. While CLK expression in the brain of the $P d p 1 \varepsilon$ mutant is variable in the presence of $L D$ cycles (that is, some cells have reduced expression of CLK, but others have relatively normal expression, data not shown), it is substantially reduced in the ventral lateral neurons in DD (Fig. 4A), consistent with previous findings in Pdp1-null larvae (Cyran et al., 2003). CLK expression is also reduced in other clock neurons such as dorsal lateral neurons $\left(\mathrm{LN}_{\mathrm{d}} \mathrm{s}\right)$ and dorsal neurons $(\mathrm{DN} 1)$ (Fig. $4 A$; supplemental Fig. 2, available at www.jneurosci.org as supplemental material). In addition, expression of PER, a direct target of CLK, is also dramatically diminished in DD, and the cycling 
of PER is not discernable in mutants (Fig. $4 B$ ). However, as noted for the Pdp1-null larvae (Blau et al., 2007), there is only marginal effect of the $P d p 1^{3135}$ mutation on PER oscillations in LD (data not shown). The effect of loss of Pdp1E on the freerunning molecular clock is in contrast to results obtained with $P d p 1^{\text {RNAi }}$ or $P d p 1^{\text {DN }}$ flies (Benito et al., 2007; Lim et al., 2007). The difference may stem from residual $P D P 1$ activity in $P d p 1^{\mathrm{RNAi}}$ and $P d p 1^{\mathrm{DN}}$ flies.

Interestingly, downregulation of another CLK-regulated protein, PDF, is seen in the small ventral lateral neurons but not in the large ventral lateral neurons (Fig. 5). This is reminiscent of findings that PDF expression is severely affected only in the small ventral lateral neurons in Clk and cyc mutants (Park et al., 2000; Allada et al., 2003). Together, these results suggest that loss of $P d p 1 \varepsilon$ results in molecular phenotypes in the central clock similar to those caused by $C l k$ or $c y c$ mutation, and provide evidence that $\mathrm{PDP} 1 \varepsilon$ is indeed a major positive regulator of CLK expression in the central pacemaker cells.

Loss of Pdp1 $\varepsilon$ alters the expression of clock genes in peripheral clocks

To examine whether $C l k$ transcription is affected in $P d p 1 \varepsilon$ mutants, we measured Clk mRNA levels in whole fly heads by quantitative real-time PCR. Most circadian gene transcripts and protein products in whole heads come from the eyes, and thus fly head assays are presumed to reflect the function of peripheral clocks. Clk mRNA levels are slightly reduced in LD and DD (Fig. 6A, B). We also assayed CLK protein levels in fly heads by Western blot analysis. In the presence of LD cycles, there is a small reduction of CLK protein levels in $P d p 1 \varepsilon$ mutants, but the CLK protein still displays cyclic mobility shifts over the course of the day (Fig. 6C). However, a more severe phenotype is observed in DD. Cyclic mobility shifts of CLK are dampened in DD in both wild type and $P d p 1^{3135}$ mutants, but the mutant, in addition, shows a reduction in the low mobility forms of CLK (Fig. 6D). Decreased mobility of CLK is thought to arise from phosphorylation, which affects its transcriptional activity (Kim and Edery, 2006; Yu et al., 2006). An effect of the $P d p 1^{3135}$ mutation on the levels and the phosphorylation of CLK suggest that CLK activity is altered in multiple ways in $P d p 1^{3135}$ mutants.

Since altered activity of CLK is predicted to affect oscillations of its targets, we also assayed the expression of genes regulated by CLK. mRNA levels of per are reduced in Pdp1e mutants (Fig. 6A). In addition, Western blot analysis revealed that peak levels of PER protein are reduced in $P d p 1^{3135}$ mutants at late night (ZT20 and
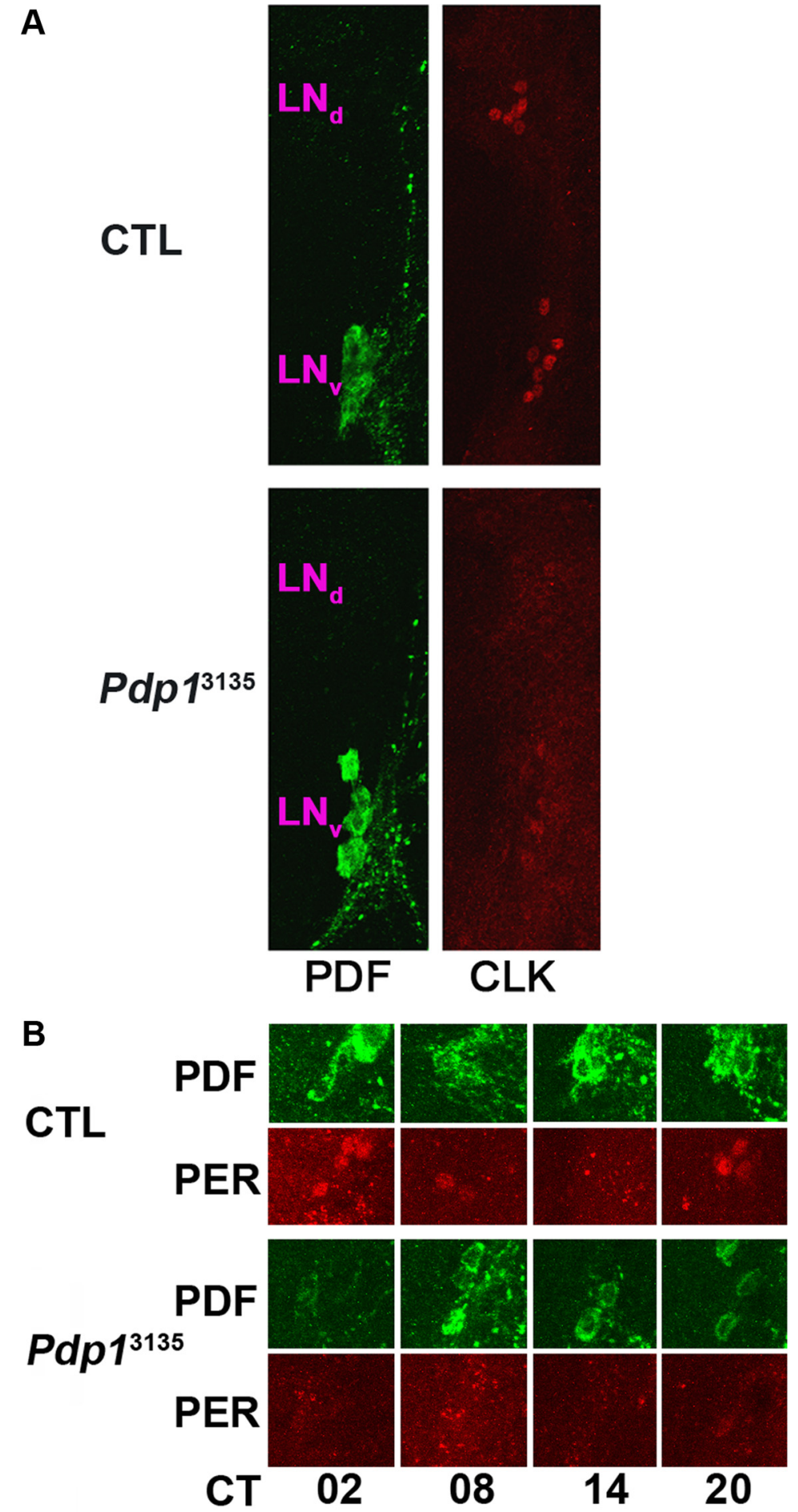

Figure 4. Circadian clock protein expression is reduced in the central clock cells of the $P d p 1^{3135}$ mutants. $\boldsymbol{A}$, CLK protein levels at CT09 are reduced in both the dorsal and ventral lateral neurons of the $P d p 1^{3135}$ mutants. $B$, PER protein expression levels are low or undetectable in most lateral neurons of $P d p 1^{3135}$ mutants. In a minority of $P d p 1^{3135}$ brain hemispheres ( 3 of 10$), 1-2$ of the small lateral neurons have subnormal levels of PER expression at CTO2. Most brain samples from Pdp $1^{3135}$ mutants have low to undetectable PDF expression in the small ventral lateral neurons. For the $P d p 1^{3135}$ mutants, samples with visible PDF in the small lateral neurons are shown, and PDF signals were enhanced to visualize the cell bodies. CTL, Background control for Pdp ${ }^{3135}$.
CT20), although the cyclic expression and phosphorylation pattern of PER are not dramatically changed in LD or DD (Fig. $6 B, D)$. Moreover, cyclic expression of a per-luciferase reporter in whole flies dampens rapidly in $P d p 1 \varepsilon$ mutant flies (Fig. 6E). Although these data support the idea that CLK activity is affected, it 


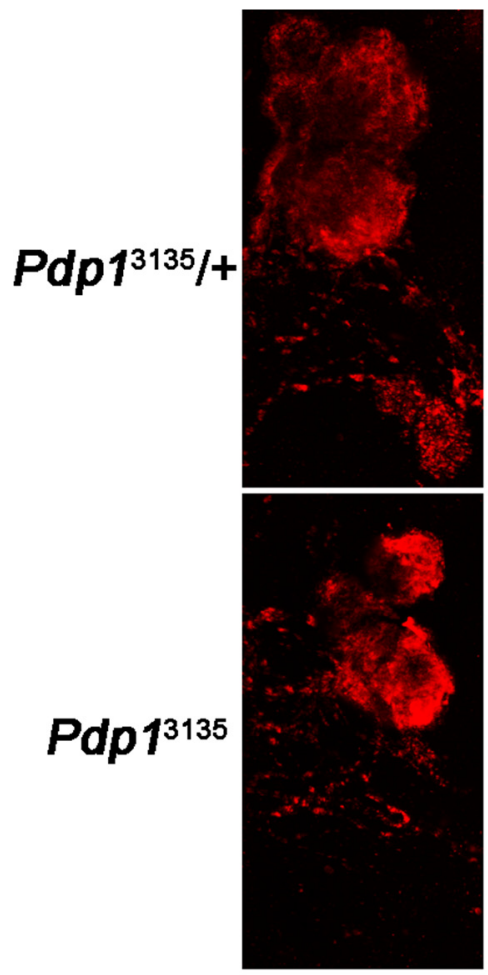

PDF

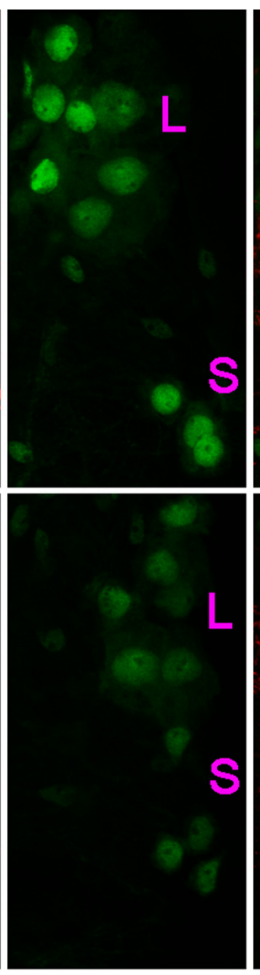

TUG>GFP

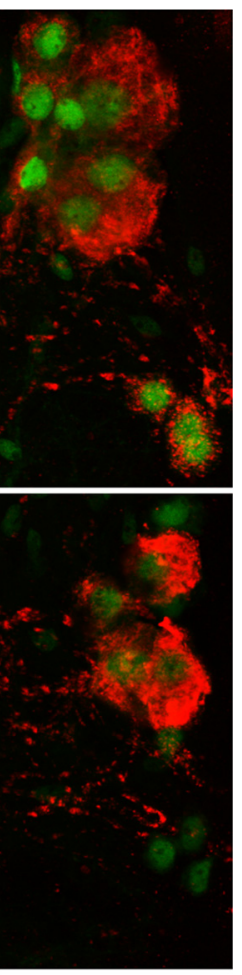

Merge
Figure 5. PDF expression is reduced in the small ventral lateral neurons of the $P d p 1^{3135}$ mutants. Cell bodies of the lateral neurons were visualized by expression of UAS-gfp driven by TUG. Representative images of four brains examined at CT21 are shown.
A

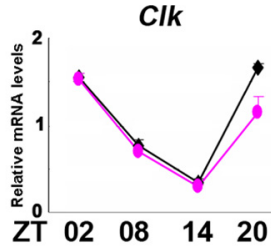

B

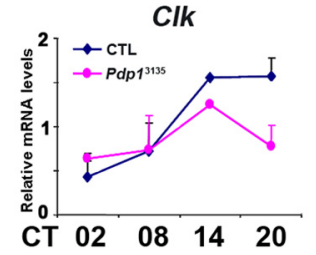

D

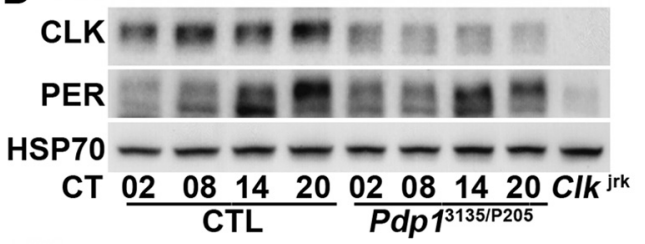

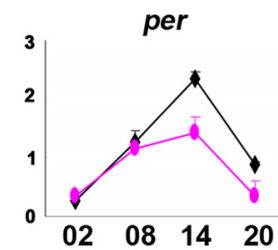

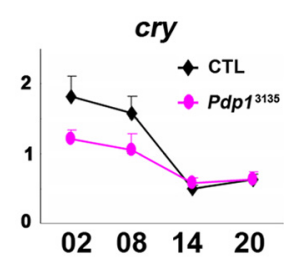

C

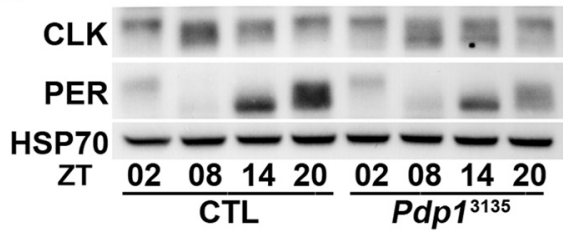




\section{cry-Gal4/+;Pdp1 $1^{3135}$}
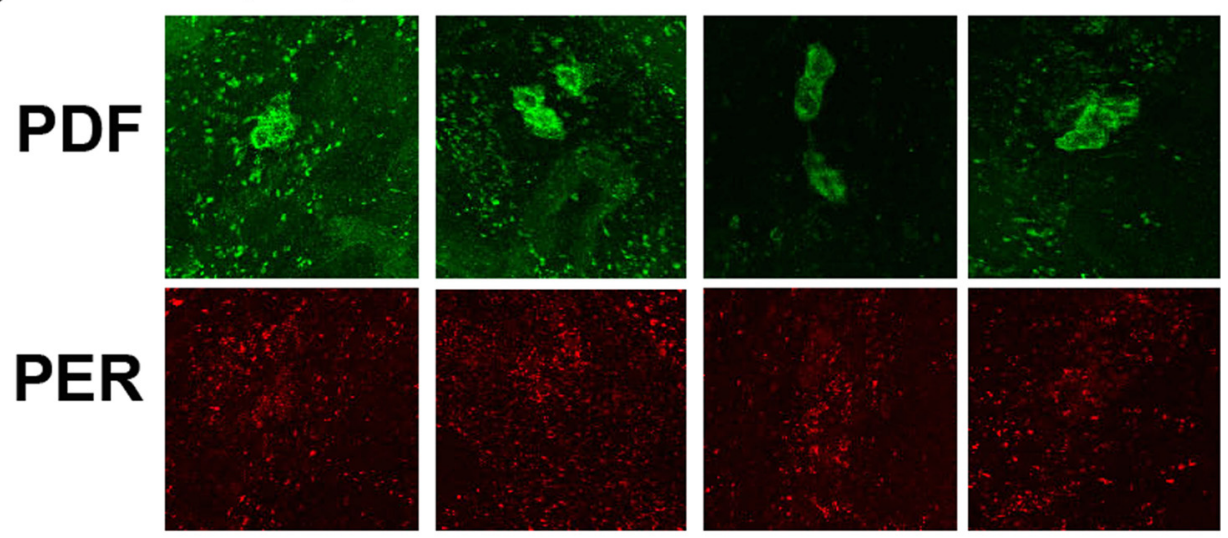

\section{cry-Gal4/UAS-Clk;Pdp13135}
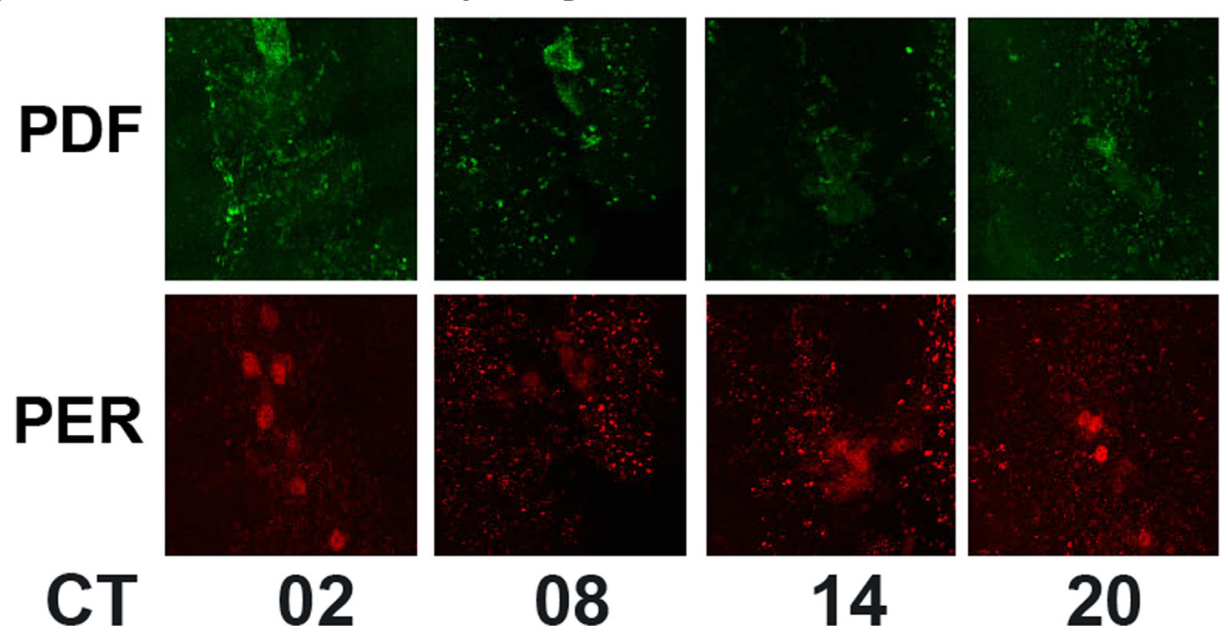

08

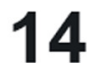

20

Figure 7. Forced expression of $C l k$ restores expression of PER in the lateral neurons of $P d p 1^{3135}$ mutants. While PER is constantly low in the $P d p 1^{3135}$ mutants, overexpression of $C l k$ driven by cry-Gal4 (line 24) elevated PER expression and restored its cycling in the lateral neurons. Note that PDF labels the large $L N_{v} s$, but it is low to undetectable in the $s m a l l \mid L_{v} s$ of $P d p 7^{3135}$ mutants. Thus a larger area is shown to cover the area where the small $\mathrm{LN}_{v} s$ normally localize. In contrast to PER, PDF expression is not restored by forced expression of $C l k$; instead, it is further reduced in the $L N_{v} s$. Representative images of 5 brains examined for each genotype and time point are shown.

Table 2. Expression of circadian clock gene Clk, per, or Pdf does not rescue the behavioral phenotype of $P d p 1^{3135}$ mutant

\begin{tabular}{|c|c|c|c|}
\hline Genotype & $n$ & $R \%$ & Period (h) \pm SEM \\
\hline cry24-G/Y;:Pdp $1^{3135} / \mathrm{TM} 3$ & 8 & 100 & $25.99 \pm 0.174$ \\
\hline cry24-G/Y;UAS-CIK/+;Pdp1 $1^{3135 / T M 3}$ & 4 & 75 & $22.80 \pm 0.312$ \\
\hline cry24-G/Y;UAS-Clk/+;Pdp1 $1^{3135}$ & 30 & 0 & \\
\hline cry24-G/yw;:Pdp1 ${ }^{3135}$ & 48 & 0 & \\
\hline cry24-G/yw;UAS-CIk;Pdp $1^{3135}$ & 41 & 0 & \\
\hline cry24-G/Y;UAS-per/+;Pdp1 $1^{3135} /$ TM3 & 23 & 74 & $28.12 \pm 0.221$ \\
\hline cry24-G/Y;UAS-per/+;Pdp1 $1^{3135}$ & 39 & 0 & \\
\hline w/Y;TUG/UAS-per;Pdp $1^{3135}$ & 15 & 0 & \\
\hline cry24-G/Y;UAS-Pdf/+;Pdp1 $1^{3135} /$ TM3 & 14 & 100 & $25.18 \pm 0.200$ \\
\hline cry24-G/Y;UAS-Pdf/+;Pdp1 $1^{3135}$ & 19 & 15 & $23.75 \pm 1.630$ \\
\hline
\end{tabular}

cry24-G: cry-Gal4 (line 24); TUG: tim-UAS-Gal4; UAS-per: UAS-per transgene (line 2-4).

nate gene expression. We have identified an $\varepsilon$-specific mutation which allows us to unequivocally establish PDP1 1 as an important component of the central clock.

PDP1 $\varepsilon$ affects CLK and PER expression in central and peripheral clocks

The mutation we discovered is a 4 bp deletion in the second exon of $P d p 1 \varepsilon$ that is predicted to introduce a frame shift and result in no functional PDP1 $\varepsilon$ protein. Loss of $P d p 1 \varepsilon$ has a strong effect on clock protein expression in the central pacemaker cells. We found that CLK levels are reduced in the lateral neurons of $P d p 1^{3135}$ mutants. Expression of PER is also dramatically reduced, demonstrating that PDP1e is an important positive regulator of the central circadian clock.

Analysis of adult heads, which are representative of peripheral clocks, demonstrates that although the effect of the $P d p 1^{3135}$ mutation on CLK levels is small, the mutation decreases the phosphorylation of CLK. Surprisingly, there is a stronger effect on per levels, which may be indicative of a CLK-independent effect of PDP1 on per. In fact, the effect on CLK phosphorylation may be secondary to the effect on PER. PER is known to promote CLK phosphorylation by "delivering" the kinase DBT (Kim and Edery, 2006; Yu et al., 2006). While CLK is constantly present in the nucleus (Houl et al., 2006), PER only enters the nucleus during the late night; thus a small reduction of PER expression at that stage might account for the reduced PER-dependent phosphorylation of CLK (Kim and Edery, 2006; Yu et al., 2006) in $P d p 1^{3135}$ mutants. Alternatively, PDP1 may regulate the expression of a kinase that phosphorylates CLK.

In general, the phenotype in peripheral clocks is weaker than that observed in central clock cells, with the exception of the 
defect in CLK phosphorylation which would not be detected through the immunofluorescence assays used to study the central clock. We assume that this difference is due to the presence of other isoforms of PDP1 in peripheral clocks. While we find that $P d p 1 \varepsilon$ is the major isoform expressed in the central clock cells, other isoforms of $P d p 1$ may be more abundant in the head and body, although we were not able to reliably detect other isoforms in Western blot assays. Transcripts of other isoforms do not cycle (Cyran et al., 2003), but they may still be able to activate Clk expression, and cycling of other clock components such as VRI may be sufficient to drive rhythmic Clk expression. Our finding that the behavioral phenotype of the pan-isoform, hypomorphic $P d p 1^{\mathrm{d} 11071}$ homozygotes is more severe than that of transheterozygous $P d p 1^{\mathrm{d} 11071 / 3135}$ flies is consistent with the possibility that non- $\varepsilon$ isoforms of $P d p 1$ function in the circadian oscillator (Table 1).

\section{Differential effects of PDP1 on molecular and behavioral rhythms}

All molecular effects of the $P d p 1^{3135}$ mutant are more pronounced in $\mathrm{DD}$ than in $\mathrm{LD}$, suggesting that factors other than Pdp1 account for the molecular cycling in LD conditions. Thus, PDP1 likely functions in the clock predominantly under freerunning conditions. However, the mutant flies are arrhythmic in LD cycles. While this is similar to, although perhaps stronger than, the LD phenotype reported for Clk and $c y c$ mutants, it is not a general feature of clock mutants. For example, per and tim mutants are rhythmic in LD cycles. Rhythms in LD arise either from entrainment of the clock to the light:dark cycle or, in the absence of a functional clock, from a process known as "masking." The arrhythmic LD behavior of $P d p 1$ mutants suggests that in addition to affecting clock function, they have defects in masking.

\section{Effect of PDP1 on PDF expression}

CLK-CYC heterodimers activate $P d f$ expression through an unknown mechanism (Park et al., 2000). Both Pdf RNA and PDF protein levels are extremely low in Clk and cyc mutants, and this reduction is more pronounced in the small lateral neurons relative to the large lateral neurons (Park et al., 2000; Allada et al., 2003). This is consistent with our finding that in $P d p 1^{3135} \mathrm{mu}-$ tants, the reduction of CLK expression is associated with reduced PDF expression in the small lateral neurons of $P d p 1^{3135}$ mutants. These data suggest that different clock cells have different sensitivity to loss of PDP1 and CLK.

Interestingly, expression of CLK with a heterologous promoter does not rescue PDF expression in $P d p 1$ mutants. This may indicate a circadian clock-independent effect of PDP1 on PDF, but we cannot rule out an alternative explanation involving levels of VRI. VRI overexpressing flies also show reduced PDF, most likely due to a posttranscriptional effect of VRI on PDF (Blau and Young, 1999). Since CLK activates vri expression, forced expression of CLK in the $P d p 1^{3135}$ mutant background may increase VRI, leading to a further reduction of PDF. Most likely, it is this effect on PDF that accounts for the failure of CLK to restore behavioral rhythms in Pdp1 mutants. In a wild-type background, CLK overexpression does not cause arrhythmia, probably because both VRI and PDP are activated simultaneously and have opposing effects. Indeed, VRI overexpression (Blau and Young, 1999) and PDP1 1 loss of function have the same effect on PDF expression.

Based upon the discussion above, it appears that the effect of PDP1 on PDF is not strictly through CLK. Thus, in this sense PDP1 has a more direct role in clock output (if PDF may be considered a clock output). In addition, it clearly has an essential role in the central clock. However, while these data establish a clock function for PDP1, they do not address the importance of the second feedback loop which drives the cyclic expression of $C l k$. PDP1 is required because it activates the expression of an essential clock gene, $C l k$, and not necessarily because it drives $C l k$ cycling. An investigation of the importance of the second feedback loop must await analysis of mutations in the negative regulator of that loop, vri.

\section{References}

Allada R, Kadener S, Nandakumar N, Rosbash M (2003) A recessive mutant of Drosophila Clock reveals a role in circadian rhythm amplitude. EMBO J 22:3367-3375.

Benito J, Zheng H, Hardin PE (2007) PDP1 $\varepsilon$ functions downstream of the circadian oscillator to mediate behavioral rhythms. J Neurosci 27:2539-2547.

Blau J, Young MW (1999) Cycling vrille expression is required for a functional Drosophila clock. Cell 99:661-671.

Blau J, Blanchard F, Collins B, Dahdal D, Knowles A, Mizrak D, Ruben M (2007) What is there left to learn about the Drosophila clock? Cold Spring Harb Symp Quant Biol 72:243-250.

Cyran SA, Buchsbaum AM, Reddy KL, Lin M-C, Glossop NRJ, Hardin PE, Young MW, Storti RV, Blau J (2003) vrille, Pdp1, and dClock form a second feedback loop in the Drosophila circadian clock. Cell 112:329-341.

Glossop NRJ, Houl JH, Zheng H, Ng FS, Dudek SM, Hardin PE (2003) VRILLE feeds back to control circadian transcription of clock in the Drosophila circadian oscillator. Neuron 37:249-261.

Hardin PE (2005) The circadian timekeeping system of Drosophila. Curr Biol 15:R714-R722.

Houl JH, Yu W, Dudek SM, Hardin PE (2006) Drosophila CLOCK is constitutively expressed in circadian oscillator and non-oscillator cells. J Biol Rhythms 21:93-103.

Kim EY, Edery I (2006) Balance between DBT/CKI $\varepsilon$ kinase and protein phosphatase activities regulate phosphorylation and stability of Drosophila CLOCK protein. Proc Natl Acad Sci U S A 103:6178-6183.

Koundakjian EJ, Cowan DM, Hardy RW, Becker AH (2004) The Zuker collection: a resource for the analysis of autosomal gene function in Drosophila melanogaster. Genetics 167:203-206.

Lee C, Bae K, Edery I (1998) The Drosophila CLOCK protein undergoes daily rhythms in abundance, phosphorylation, and interactions with the PER-TIM complex. Neuron 21:857-867.

Lim C, Lee J, Koo E, Choe J (2007) Targeted inhibition of Pdp1 epsilon abolishes the circadian behavior of Drosophila melanogaster. Biochem Biophys Res Commun 364:294-300.

Mitsui S, Yamaguchi S, Matsuo T, Ishida Y, Okamura H (2001) Antagonistic role of E4BP4 and PAR proteins in the circadian oscillatory mechanism. Genes Dev 15:995-1006.

Park JH, Helfrich-Förster C, Lee G, Liu L, Rosbash M, Hall JC (2000) Differential regulation of circadian pacemaker output by separate clock genes in Drosophila. Proc Natl Acad Sci U S A 97:3608-3613.

Peng Y, Stoleru D, Levine JD, Hall JC, Rosbash M (2003) Drosophila freerunning rhythms require intercellular communication. PLoS Biol 1:E13.

Reddy KL, Wohlwill A, Dzitoeva S, Lin M-H, Holbrook S, Storti RV (2000) The Drosophila PAR Domain Protein 1 ( $P d p 1)$ gene encodes multiple differentially expressed $\mathrm{mRNAs}$ and proteins through the use of multiple enhancers and promoters. Dev Biol 224:401-414.

Reddy KL, Rovani MK, Wohlwill A, Katzen A, Storti RV (2006) The Drosophila Par domain protein I gene, $P d p 1$, is a regulator of larval growth, mitosis and endoreplication. Dev Biol 289:100-114.

Rubin GM, Spradling AC (1982) Genetic transformation of Drosophila with transposable element vectors. Science 218:348-353.

Sathyanarayanan S, Zheng X, Xiao R, Sehgal A (2004) Posttranslational regulation of Drosophila PERIOD protein by protein phosphatase 2A. Cell 116:603-615.

Wu MN, Koh K, Yue Z, Joiner WJ, Sehgal A (2008) A genetic screen for sleep and circadian mutants reveals mechanisms underlying regulation of sleep in Drosophila. Sleep 31:465-472.

Yu W, Zheng H, Houl JH, Dauwalder B, Hardin PE (2006) PER-dependent rhythms in CLK phosphorylation and E-box binding regulate circadian transcription. Genes Dev 20:723-733. 